\title{
La mejora del rendimiento académico de estudiantes universitarios inmigrantes: La importancia del estudio en grupo ${ }^{1}$
}

\section{Karlos La Serna Studzinski}

Universidad del Pacífico

laserna_k@up.edu.pe

Recibido: 13/04/2019

Aprobado: 04/06/2020

1. El presente artículo se basa en parte de los hallazgos de la tesis de maestría en Sociología del autor, titulada "El viaje del héroe: La cartografía de pruebas de los 'estudiantes de provincia' de la Universidad del Pacífico". Dicha tesis está disponible en http://tesis.pucp.edu.pe/repositorio/handle/20.500.12404/13880 


\title{
La mejora del rendimiento académico de estudiantes universitarios inmigrantes: La importancia del estudio en grupo
}

\section{Resumen}

El presente artículo parte de los resultados de modelos econométricos que permitieron identificar una brecha en el rendimiento académico entre los estudiantes inmigrantes de la Universidad del Pacífico y sus pares de Lima cuando iniciaban sus estudios superiores. Específicamente, los estudiantes que egresaron de la educación secundaria en Lima mostraban un mejor rendimiento que sus pares de otras regiones. Dicha brecha se reducía conforme transcurrían los semestres. Estos resultados motivaron una investigación cualitativa que acudió a entrevistas a profundidad para conocer la experiencia universitaria de los estudiantes migrantes. De este modo, fue posible identificar que sus problemas académicos eran originados por déficits que arrastraban de su etapa escolar: la costumbre de estudiar individualmente y tan solo unas pocas horas el día previo a las evaluaciones; la falta de conocimientos profundos de Matemáticas; $y$, finalmente, la falta de dominio del idioma inglés. Para superar dichas desventajas y mejorar su rendimiento académico, la mayoría de entrevistados descubrió que la estrategia que mejor funcionaba era estudiar en grupos.

Palabras clave: Inmigración, universidad, plan de estudios, rendimiento académico, métodos de estudio

\section{Improving the academic performance of immigrant university students: The importance of group study}

\begin{abstract}
The present article summarizes the results of econometric models, which allowed the identification of a gap in academic performance among students of Universidad del Pacífico when they started higher education. Specifically, students who finished high school in Lima had better academic performance than their peers from other regions (non-limeños). This gap narrowed as the semesters passed. Motivated by these results, we carry out a qualitative research and conducted in-depth interviews in order to learn about the university experience of immigrant students. We identify that academic difficulties had its origin in non-limeños' deficits brought from their high-school stage: habits of studying individually and only a few hours before evaluations; lack of deep Mathematics knowledge; and, finally, the lack of English proficiency. To overcome these disadvantages and improve their academic performance, the majority found out group study as the best strategy.

Keywords: Immigration, university, curriculum, academic performance, study methods
\end{abstract}




\section{Introducción}

Desde la década de 1980, en Latinoamérica, el número de universidades se ha incrementado significativamente. Según Rama (2005, p. 50), este proceso también se expresó en la expansión de las universidades localizadas en las grandes ciudades o en las capitales, a través de sedes que fueron ubicadas en las diversas regiones de cada país. Esta expansión de las universidades contribuyó a reducir las fuertes migraciones estudiantiles desde las regiones hacia las capitales nacionales.

En el caso peruano, la matrícula despegó en Lima y en otras provincias, producto de la liberalización del mercado educativo universitario desarrollada durante la década de 1990 (Cuenca, 2015, p. 11). Sin embargo, la comparación de los resultados de los dos únicos Censos Nacionales Universitarios realizados en el Perú evidencia que la presencia de los alumnos inmigrantes - usualmente, calificados como "los estudiantes de provincia" - se ha incrementado en las universidades privadas del departamento de Lima, donde se ubica la capital peruana (Tabla 1).

Tabla 1. Estudiantes inmigrantes ${ }^{2}$ en universidades del departamento de Lima $^{3}$

\begin{tabular}{cccc}
\hline Año & Públicas & Privadas & Total \\
\hline 1996 & $27,6 \%$ & $24,0 \%$ & $25,5 \%$ \\
2010 & $26,8 \%$ & $39,7 \%$ & $36,2 \%$ \\
\hline
\end{tabular}

Fuente: Instituto Nacional de Estadística e Informática (1997) e Instituto Nacional de Estadística e Informática y Asamblea Nacional de Rectores (2011)

Debido al crecimiento de los estudiantes inmigrantes en las universidades privadas del departamento de Lima, es pertinente analizar los desafíos que enfrentan estos alumnos. Ciertamente, en el Perú, pocas investigaciones se han dedicado a este tipo de análisis. Por ejemplo, Pereyra-Elías et al. (2010) evaluaron la prevalencia de síntomas depresivos y sus factores asociados en los estudiantes de la Facultad de Ciencias de la Salud de la Universidad Peruana de Ciencias Aplicadas (UPC). Si bien estos autores supusieron que la migración estudiantil era uno de los factores asociados a síntomas depresivos, en el caso

2. Para determinar si un alumno que seguía estudios en una universidad limeña era inmigrante, fue identificado el departamento de nacimiento del alumno. Cuando el departamento de nacimiento no era Lima, el alumno fue considerado como "inmigrante".

3. En el caso de la Universidad del Pacífico, que constituye el ámbito de estudio de la investigación en la cual se basa el presente artículo, el porcentaje estudiantes inmigrantes se incrementó de $15,7 \%$ a 17,9\% entre los años 1996 y 2010. 
de los estudiantes inmigrantes que encuestaron (19,6\% de los encuestados), sus resultados no confirmaron dicha hipótesis. Por ello, plantearon la posibilidad de que la influencia de la variable inmigración sobre los síntomas depresivos era atenuada por las características de la población estudiada (nivel socioeconómico alto y oportunidad de estudiar en Lima), así como por el Programa de Acompañamiento Universitario brindado por la UPC para apoyar a los inmigrantes.

Por su parte, García y Medina (2011) estudiaron a una muestra de cien alumnos de la Pontificia Universidad Católica del Perú (PUCP) procedentes de departamentos del Perú diferentes de Lima, mediante una investigación cuantitativa-cualitativa exploratoria. Sus hallazgos mostraron que

[e]l proceso de integración del alumno de provincia que recién ingresa a la Universidad presenta características diferentes a la de aquel que reside en Lima, pues no sólo debe adaptarse a sus propios cambios que va experimentando y a su nueva forma de ver el mundo sino que tiene que redefinir sus roles y competencias en un nuevo ambiente familiar, académico y de grupos de pares que pasan a tener una influencia importante en su desenvolvimiento universitario constituyéndose esta etapa además de crítica, de vulnerabilidad relativa. (García y Medina, 2011, p. 128)

La investigación de García y Medina (2011) evidencia que los estudiantes inmigrantes enfrentan desafíos particulares debido a la salida de sus localidades de origen, los cuales se suman a los que tiene todo estudiante universitario. Las autoras encontraron que, a pesar de los mayores obstáculos que debían enfrentar, varios de los estudiantes inmigrantes de la PUCP alcanzaron resultados académicos satisfactorios, y superaron las dificultades, adversidades y sentimientos de soledad (García y Medina, 2011, p. 2). Estos estudiantes destacaron por sus anhelos de desarrollo personal, así como por su deseo de no defraudar a sus padres y, en general, de contribuir a mejorar los niveles de vida de sus familias residentes en sus regiones de origen. Las autoras también destacaron la importancia del apoyo brindado a dichos estudiantes por el Proyecto "Integrándome a la Vida Universitaria", ejecutado por el Servicio de Promoción y Apoyo Social de la PUCP.

Una limitación de la investigación de García y Medina (2011) es que basó parte importante de sus conclusiones en estadística descriptiva, la cual no permite determinar, con seguridad, la relación de causalidad existente entre las variables estudiadas. Según La Serna (2011, p. 32), esta última limitación puede ser superada mediante los modelos econométricos que suelen aplicar las investigaciones de los economistas. Dichos modelos sí permiten establecer el efecto de una variable independiente sobre una variable dependiente, en presencia de un conjunto de otras variables o controles que ajustan el primer efecto mencionado a fin de que refleje exclusivamente la influencia de la variable 246 I independiente sobre la dependiente. 
Precisamente, Beltrán y La Serna (2011) acudieron a modelos econométricos de panel - los cuales siguen a un grupo de individuos a lo largo del tiempo- para identificar los principales factores académicos, psicológicos, sociofamiliares y de identificación (edad y sexo) que explican la evolución del rendimiento académico de los estudiantes que ingresaron a la Universidad del Pacífico (UP) en el año 2006. El período de observación de su estudio abarcó desde el semestre 2006-I hasta el 2009-I, incluidos los ciclos de los veranos 2007, 2008 y 2009. En otras palabras, comprendió un total de diez ciclos académicos: siete regulares y tres de verano (Beltrán y La Serna, 2011, p. 252).

Los autores usaron como variables dependientes la calificación acumulada anual y la calificación acumulada semestral de los estudiantes, a partir de las cuales estimaron dos ecuaciones (modelos). Cada una de ellas relacionaba una de las dos variables dependientes con factores académicos, psicológicos, sociofamiliares y de identificación. Uno de los resultados que arrojó el primer modelo es que, durante su primer año de estudios, los alumnos procedentes de provincias diferentes a Lima (estudiantes inmigrantes de aquí en adelante) obtenían en promedio 0,52 puntos menos de calificación acumulada anual que sus pares de Lima. Sin embargo, el impacto negativo de la migración decrecía con el tiempo; específicamente, la brecha académica se reducía durante el segundo año de estudios. En términos precisos, la diferencia de calificación acumulada anual entre los limeños y los inmigrantes caía en 0,16 puntos (Beltrán y La Serna, 2011, pp. 287-288).

Además, mediante el modelo explicativo de la calificación acumulada semestral, Beltrán y La Serna (2011, p. 294) hallaron que los estudiantes inmigrantes obtenían en promedio 0,48 puntos menos de calificación acumulada que el resto durante el primer semestre, pero esta brecha se reducía al finalizar el sexto semestre de estudios. En términos precisos, la diferencia de calificación acumulada semestral entre los limeños y los inmigrantes caía en 0,14 puntos.

En suma, Beltrán y La Serna (2011) encontraron evidencia, en la Universidad del Pacífico, de que los alumnos inmigrantes obtenían un menor rendimiento académico que sus pares de Lima al iniciar sus estudios universitarios, pero que dicha brecha se reducía conforme transcurrían los semestres. A partir de ello, el estudio planteó como hipótesis que los costos que genera la pérdida de la red de protección social que la familia provee - como resultado de la migración desde las provincias para estudiar en la capital- reducirían su importancia una vez que el estudiante lograra ambientarse adecuadamente a la ciudad de Lima y desarrollara una red de protección social específica en ella (Beltrán y La Serna, 2011, pp. 287-288).

Lamentablemente, la naturaleza de los métodos econométricos aplicados no permitía confirmar la hipótesis previa de Beltrán y La Serna ni identificar cuál o cuáles eran las explicaciones pedagógicas del menor rendimiento académico que inicialmente mostraban los estudiantes inmigrantes. Los métodos econométricos tampoco permitieron identificar cuáles fueron las estrategias que usaron los alumnos inmigrantes para mejorar su rendimiento académico. 
Frente a estos dos dilemas, el presente artículo partió de los resultados de los modelos de Beltrán y La Serna (2011), los cuales fueron profundizados e interpretados mediante métodos cualitativos usuales en la investigación educativa. Tal como detalla la siguiente sección, se aplicaron entrevistas a profundidad que permitieron recoger la experiencia de inserción a la vida universitaria de los estudiantes inmigrantes que pertenecen a la cohorte estudiada por Beltrán y La Serna (2011). De este modo, se respondió las dos preguntas previamente planteadas.

\section{Métodos de investigación y proceso de levantamiento de datos}

Para interpretar los resultados de Beltrán y La Serna (2011), se planeó un diseño cualitativo. De este modo, fueron analizados, desde la experiencia de los estudiantes inmigrantes de la Universidad del Pacífico, los desafíos que enfrentaron para insertarse a la vida universitaria, en especial, pero no únicamente, los desafíos académicos. Para dicho análisis, fue usado como operador analítico el modelo de las pruebas al que han acudido diversos sociólogos educativos como Anne Barrère (2016) y Danilo Martuccelli (2004). En términos de Araujo y Martuccelli, las pruebas son "[...] desafíos históricos, socialmente producidos, culturalmente representados, desigualmente distribuidos que los individuos están obligados a enfrentar en el seno de un proceso estructural de individuación" (2010, p. 83).

La investigación también abordó el proceso de mejora del rendimiento académico de los estudiantes inmigrantes. Así, la unidad de análisis fueron los alumnos inmigrantes de la población estudiada por Beltrán y La Serna (2011). Dicha población abarcaba 474 estudiantes que ingresaron a la Universidad del Pacífico durante el año 2006, de los cuales 43 eran inmigrantes. Esta última condición era asignada cuando el colegio donde el estudiante de la UP terminó la educación secundaria estaba ubicado en alguna provincia diferente de Lima. Conviene destacar que, de los 43 alumnos inmigrantes, 4 no concluyeron sus estudios superiores en la Universidad del Pacífico; en algunos casos, porque abandonaron los estudios por voluntad propia, en otros, debido a que su bajo rendimiento académico determinó que sean separados de la Universidad.

Los datos sobre los desafíos académicos de los estudiantes inmigrantes fueron levantados mediante entrevistas semiestructuradas y a profundidad. Para este proceso, en primer lugar, fue realizado un piloto de tres entrevistas a estudiantes inmigrantes. Dichas entrevistas, realizadas a inicios de julio del año 2016, permitieron mejorar la guía de entrevistas para el trabajo de campo principal. Este último fue desarrollado entre abril y junio del año 2017. Como resultado de esta etapa, fueron entrevistadas 19 personas, que pertenecían al grupo de los 43 estudiantes inmigrantes que iniciaron estudios universitarios en la UP durante el año 2006. Dos de ellas formaban parte del grupo de cuatro estudiantes inmigrantes que no concluyeron sus estudios en la Universidad del 
Pacífico ${ }^{4}$, pero egresaron de otra universidad y, al igual que los demás entrevistados, se encontraban laborando al momento de ser entrevistados. Es decir, todos los entrevistados ya habían egresado de sus estudios de bachillerato.

Conviene destacar que fueron realizadas entrevistas a profundidad hasta alcanzar el criterio o principio de saturación asociado a los métodos cualitativos; es decir, siguieron siendo incorporados entrevistados hasta que la información que proveían comenzó a repetirse sistemáticamente. En ese sentido, se respetó la regla que sugieren Hernández, Fernández y Baptista (2010, p. 459), según la cual es necesario levantar información hasta que los datos comienzan a ser "repetitivos" o redundantes, y los nuevos análisis confirmen aquello que ya ha sido fundamentado.

Además, para triangular la información generada, fueron realizadas tres entrevistas a personal administrativo de la Universidad del Pacífico que había laborado en el período en el cual los miembros de la población objeto de estudio eran alumnos. Se trató de una asistenta social y dos psicólogos de la UP. Dichas funciones determinaban que hayan estado relacionados con la problemática académica de los estudiantes inmigrantes. Para desarrollar las entrevistas al personal administrativo, al inicio, se les planteó un resumen de los hallazgos y, después, se les pidió su opinión con respecto de estos últimos.

\section{Resultados}

En la presente sección, son descritos los resultados de la investigación. Para ello, primero, son identificados los desafíos académicos que enfrentaron los alumnos inmigrantes entrevistados y, luego, son explicadas las estrategias que desarrollaron para superar dichos desafíos.

\section{Los desafíos académicos}

En general, es posible afirmar que el currículo ${ }^{5}$ de la Universidad del Pacífico demandó ciertos conocimientos y experiencias previas (saberes conceptuales, habilidades y actitudes) que no poseían los estudiantes inmigrantes, al menos en la profundidad necesaria. Esto calza con las ideas del movimiento constructivista, el cual reconoce que el aprendizaje demanda una sólida base de saberes previos. Precisamente, Ausubel (1976, pp. 40-41) planteó que un "aprendizaje significativo" sucede si la tarea de aprendizaje puede relacionarse de modo no arbitrario con lo que el alumno ya sabe. En otros términos, el

4. Es oportuno mencionar que las entrevistas realizadas a quienes no concluyeron sus estudios en la UP sirvieron como mecanismo de control de los hallazgos, específicamente, para aproximarse a las experiencias de quienes no concretaron el proceso de integración a la vida universitaria en la UP.

5. En este caso, el currículo es entendido como el "plan de formación" (Becerra y La Serna, 2016, p. 46). 
aprendizaje significativo ocurre cuando los saberes nuevos se relacionan con los saberes previos.

Desde la experiencia de los estudiantes inmigrantes entrevistados, entre los saberes previos demandados por el currículo de la Universidad del Pacífico, en primer lugar, se encuentran métodos de estudio. En efecto, el método de estudio que típicamente usaban los entrevistados en el colegio, caracterizado por ser individual, memorístico y desarrollado el día previo a la evaluación, no funcionó en la Universidad por el nivel de dificultad de las evaluaciones. En la mayoría de las asignaturas universitarias que cursaron, aprender de memoria - tal como solían hacer durante la etapa escolar - no era suficiente, debido a que las preguntas de las evaluaciones de la Universidad les demandaban interpretar información a la luz de la teoría o aplicarla a diversos contextos, así como brindar respuestas con un sentido crítico o interpretativo. Adicionalmente, a diferencia de lo que sucedía en sus colegios, en las evaluaciones de los cursos universitarios con contenidos cuantitativos, no era común encontrar preguntas que se parecían a las que habían sido resueltas en clase. Evidentemente, la falta de "preguntas tipo" exigía un aprendizaje más "profundo" de los contenidos enseñados.

Aquí conviene reconocer que el aprendizaje profundo es derivado de la "necesidad sentida" de abordar las tareas académicas de forma adecuada y significativa, de manera que el estudiante trate de utilizar las actividades cognitivas más apropiadas para desarrollarlas (Biggs, 2006, p. 35). Este enfoque de aprendizaje ocurre

cuando los estudiantes sienten esta necesidad de saber, procuran centrarse en el significado subyacente: en las ideas principales, temas, principios o aplicaciones satisfactorias. Esto requiere un sólido fundamento de conocimientos previos relevantes, de manera que los estudiantes que necesitan saber tratan naturalmente de aprender los detalles, así como de asegurarse que comprenden. (Biggs, 2006, p. 35)

Ciertamente, el enfoque profundo se opone al denominado enfoque de aprendizaje "superficial", en el cual prima la intención de liberarse de la actividad académica con el mínimo esfuerzo, aunque ofreciendo la sensación de satisfacer las exigencias académicas. De este modo, el estudiante universitario acude a actividades de bajo nivel cognitivo cuando debería aplicar unas de nivel superior. Ejemplos del enfoque superficial son rellenar un informe de investigación de datos que no aportan; citar referencias secundarias como si fueran primarias; producir una relación de puntos, en lugar de construir un razonamiento; aprender "al pie de la letra" contenidos seleccionados, en vez esforzarse por el entendimiento de los mismos; etc (Biggs, 2006, p. 32). El estudiante con enfoque superficial se concentra en reproducir la información adquirida. Su intención es evitar el fracaso sin trabajar demasiado. 
para aprobar (López y López, 2013, p. 137). De hecho, las experiencias de los entrevistados revelaron que, al iniciar la universidad, aplicaban un método de estudio enmarcado en un enfoque de aprendizaje superficial que conducía a un bajo rendimiento académico, por lo cual progresivamente fueron abandonando dicho método.

El déficit escolar de saberes previos no solo se evidenció en los métodos de estudio, sino también en conocimientos de Matemáticas de nivel escolar. En ese sentido, varios de los entrevistados reclamaban que sus colegios no les habían brindado la base matemática necesaria para las demandas universitarias. Conviene reconocer que la mayoría de entrevistados cursaron una asignatura destinada a cubrir el déficit de conocimientos de Matemáticas que podrían arrastrar de la etapa escolar, denominada "Nivelación de Matemáticas". Sin embargo, incluso en esta última asignatura, enfrentaron serios problemas. Tal como manifestó una entrevistada:

[...] para mí, la verdad es que el primer año fue horrible, porque yo estaba acostumbrada a sacar buenas notas. [...] en el primer examen, me acuerdo, de Economía me saqué 4. Casi me muero. [...] en el primer ciclo me tuve que retirar de Nivelación de Matemática; jalé Economía (Entrevistada 8).

El testimonio previo evidencia que no solo los cursos de Matemáticas representaron un gran desafío, sino también otros cursos que demandaban una sólida base cuantitativa, como los del campo de la Economía. Las dificultades de estos últimos cursos se debían, en parte importante, a que muchos de los entrevistados habían cursado asignaturas escolares concentradas solo en contenidos numéricos (Matemáticas, Física, Química, etc.) o solo en contenidos del campo de las letras (Lenguaje, Literatura, Historia, etc.). Sin embargo, las evaluaciones de las asignaturas de Economía de la Universidad, muchas veces, demandan combinar comprensión lectora con cálculos.

Finalmente, en varios cursos de la UP -incluso, en asignaturas de los primeros años- los profesores encargaban lecturas redactadas en inglés. Lamentablemente, la mayoría de los entrevistados manifestó que, al iniciar sus estudios universitarios, no dominaba dicho idioma. Así, varios entrevistados sintieron una fuerte desventaja frente a sus compañeros limeños que sí dominaban el inglés.

En suma, los estudiantes inmigrantes emprendieron un proceso de aprendizaje de disciplinas que exigían una base de conocimientos previos que no poseían con la profundidad necesaria. Ciertamente, conforme pasaban los semestres, desarrollaron, por cuenta propia, estrategias que les permitieron superar los desafíos académicos que enfrentaron. 


\section{Las estrategias desarrolladas para superar los desafíos académicos}

Para superar los desafíos del currículo de la UP y mejorar así su desempeño académico, los estudiantes inmigrantes desarrollaron diversas estrategias. Una de ellas fue incrementar las horas de estudio, mientras que otra fue prepararse con varios días de anticipación para las evaluaciones de los cursos más difíciles. Otra estrategia mencionada por algunos entrevistados fue acudir a academias particulares. Se trata de equipos de profesores que no laboran en la Universidad del Pacífico, pero que ofrecen asesoría bajo el formato de clases grupales para los cursos que son considerados como los más difíciles y suelen presentar las mayores tasas de desaprobación ${ }^{6}$. Ciertamente, las limitaciones económicas determinaron que esta no fuera una estrategia mayoritariamente usada, pues las clases que eran ofrecidas en las académicas particulares implicaban un gasto adicional. Este era un costo que la mayoría de entrevistados no podía asumir, debido a que sus familias ya estaban realizando un gran esfuerzo económico para asumir todos los gastos que implicaba vivir en Lima y estudiar en la UP.

Sin embargo, lo más importante para mejorar su rendimiento académico fue cambiar el método de estudio individual y memorístico que arrastraban de la etapa escolar. En ese sentido, el peso de la evidencia empírica que recogió la presente investigación confirma que estudiar más horas no bastaba, pues la dificultad de las asignaturas les demandaba a los entrevistados contrastar los hallazgos y los aprendizajes propios con los de otros compañeros para disponer del bagaje necesario para superar el nivel de exigencia de las evaluaciones que enfrentaban. A partir de ello, la mayoría de los entrevistados descubrió que la estrategia que mejor funcionaba -especialmente, en los cursos cuantitativos- era reunirse en grupos de dos a cinco estudiantes para repasar los contenidos teóricos; resolver conjuntamente ejercicios de evaluaciones de semestres anteriores; y, así, apoyarse mutuamente, complementando sus saberes. La importancia del estudio grupal ${ }^{7}$ en la superación de los desafíos curriculares es revelada por los siguientes testimonios (a los cuales se suma un conjunto de testimonios que, por cuestión de un análisis más ordenado del material de campo, son presentados algunos párrafos más adelante):

6. Dichas academias, comúnmente denominadas "grupos de estudio", también se dedican a preparar a los postulantes para los exámenes de ingreso a la Universidad (como en una academia preuniversitaria) y a ofrecer asesoría a los estudiantes de la escuela preuniversitaria de la Universidad del Pacífico.

7. Es importante no confundir el "estudio grupal" (que también será denominado "estudio en grupo") con las clases particulares ofrecidas por los denominados "grupos de estudio", cuya naturaleza fue explicada en el párrafo anterior. 
Me di cuenta de que estudiando solo era muy difícil. Tenías que juntarte con dos o tres amigos para poder intercambiar, por ejemplo, más en cursos de números: Matemáticas, Estadísticas [...]. (Entrevistado 5)

En la UP no bastaba escuchar la clase para rendir un examen. [...] En la Universidad estudiaba en grupos. (Entrevistado 11)

En la Universidad, cambió [refiriéndose a su método de estudio]; en la Universidad estudiábamos más de manera grupal. Para los cursos de Economía, que me costaban más, normalmente mi casa siempre era el punto, porque yo era la que vivía sola. [...]. (Entrevistada 12)

Te reúnes a estudiar en grupo para los exámenes difíciles. [...] Al inicio estudiaba sola. [...] En algunos cursos, como Matemáticas, servía, bastaba [estudiar sola], pero por ahí que, en algunos cursos de Economía, después ya opté por estudiar en grupo. (Entrevistada 14)

Empecé estudiando individualmente, pero de ahí pasé a estudiar, en algunos casos, grupalmente, con las cuatro personas que te digo o con cualquier otra persona de mi curso. (Entrevistada 15)

Las entrevistas revelaron que algunos estudiantes se preparaban para las evaluaciones únicamente mediante estudio grupal, en especial, cuando se trataba de los cursos que eran considerados más difíciles. En cambio, otros alumnos se preparaban individualmente, pero estudiaban de modo grupal el día previo a la evaluación, a manera de repaso. Sea como fuere, desde la experiencia de la mayoría de entrevistados, el estudio en grupo constituyó un cambio muy importante en las estrategias de aprendizaje que arrastraban de la etapa escolar y contribuyó sustancialmente a mejorar su rendimiento académico.

La importancia del estudio en grupo para mejorar el aprendizaje calza con las propuestas del constructivismo educativo, según el cual el aprendizaje es fruto de una construcción personal, pero de carácter activo. En ese sentido, para generar aprendizaje, además del individuo que aprende, también son piezas imprescindibles los "otros significativos", es decir, los agentes culturales (Solé y Coll, 1998, p. 15). Entre estos agentes culturales, están los familiares; los profesores; los amigos; y, por supuesto, los compañeros de estudio.

Ciertamente, las contribuciones al proceso de aprendizaje que ha mostrado el estudio en grupo han llevado a los especialistas en educación a promover que los profesores apliquen estrategias como el denominado "grupo de aprendizaje cooperativo". En este caso, la cooperación

consiste en trabajar juntos para alcanzar objetivos comunes. En una situación cooperativa, los individuos procuran obtener resultados que sean beneficiosos para ellos mismos y para todos los demás miembros 
del grupo. El aprendizaje cooperativo es el empleo didáctico de grupos reducidos en los que los alumnos trabajan juntos para maximizar su propio aprendizaje y el de los demás. (Johnson, Johnson y Holubec, 1999, p. 5)

Sin embargo, es importante reconocer que la conformación de los grupos de estudio que realizaron los alumnos inmigrantes no fue dirigida por los profesores ni promovida por alguna instancia de la UP; por el contrario, fue espontánea. Al respecto, en la literatura, existe cierta evidencia empírica sobre los efectos positivos de los grupos de estudio conformados espontáneamente en el ámbito de la educación superior. Precisamente, Tang (1993) encontró que los alumnos de Fisioterapia del Politécnico de Hong Kong que habían formado espontáneamente grupos de estudio tendieron a involucrarse en estrategias cognitivas de alto nivel, tales como el análisis y el establecimiento de relaciones, así como la aplicación mediante la experimentación, la discusión, la argumentación y el debate. Estos hallazgos calzan con las razones que motivaron a los alumnos inmigrantes entrevistados a estudiar en grupo. En efecto, tal como evidencian los siguientes testimonios, el intercambio de ideas, la enseñanza mutua y la consecuente complementación de sus saberes estaban entre las principales virtudes que los entrevistados le encontraban al estudio en grupo:

[...] siento que estudio mejor solo, pero sí, me juntaba de vez en cuando; a veces, cuando tenía problemas para algo o cuando me pedían. [...] [Refiriéndose a un compañero de estudios]: es bien capo, entonces, si yo no entendía algo, me ayudaba. (Entrevistado 4)

[...] otra cosa que me ayudó bastante a mí, sobre todo en Matemática y en Economía, era estudiar en grupo; porque estudiar sola, o sea, el problema no sabes cómo desarrollarlo y por más que tengas el libro quizás no puedes, pero otra persona viene y te dice "pero míralo por este lado" y lo puedes hacer, ¿no? (Entrevistada 8 )

Nos apoyábamos entre todas: algunas sabían más, otras sabían menos, etcétera; pero, entre todas, estudiábamos. Nos juntábamos en mi casa y ahí íbamos repasando las notas para cuando tuviéramos examen. (Entrevistada 12)

[Refiriéndose a los compañeros con los que estudiaba en grupo] el hecho de que tú les vayas comentando te ayuda a repasar lo que ya habías visto y a que ellos te den más. [...] a veces yo también iba no sabiendo nada, no leyendo nada y también el hecho de que ellos me contaran me ayudaba, pero adicionalmente a eso, yo aparte leía. (Entrevistada 15) 
[...] los números o, por ejemplo, Informática para los Negocios, yo no es que sentía la necesidad de hacerlo en grupo, porque también podía estudiar solo, pero también creo que era muy útil para mí y trataba de hacerlo, que era estudiar en grupo. Uno, porque, si no sabía, me enseñaban; y dos, porque si yo sabía enseñaba y creo que enseñando te pones mucho más sólido en los conocimientos, ¿no? En una de esas estás enseñando y te das cuenta de que no lo sabes [...] tan bien como creías. (Entrevistado 16)

Creo que estudiaba sola excepto el día anterior al examen, porque creo que todo el mundo estudiaba solo y el día anterior al examen nos juntábamos más como para discutir temas, algunas cosas que no entendíamos o para ponernos casos. (Entrevistada 17).

Al momento de iniciar sus estudios universitarios, los alumnos limeños ya conocían a algunos de sus compañeros de aula gracias a sus experiencias escolares o en otros espacios de sociabilidad asociados a su posición socioeconómica. Por el contrario, los estudiantes inmigrantes, debido a que constituían un grupo minoritario sin vínculos previos con pares de la ciudad de Lima, tuvieron que reconstruir sus redes sociales y, mediante un mecanismo de "ensayo y error" ir armando grupos de compañeros con los cuales sentían que era productivo estudiar. Como resultado de este proceso, parte importante de los integrantes de los grupos que armaron para estudiar estaban conformados por alumnos de otras provincias (no limeños). La razón de esta forma de agrupación se debe precisamente a que los grupos conformados por alumnos limeños eran bastante herméticos, por lo cual los estudiantes inmigrantes terminaban quedando relativamente aislados (sobre todo, en sus primeros años de estudios). Ello, a su vez, los forzó a integrarse con otros estudiantes inmigrantes (no necesariamente provenientes de su misma provincia). Esta dinámica ocurría, por ejemplo, cuando los profesores de la UP pedían que los estudiantes se organicen en grupos, ya sea para desarrollar actividades en clase o para realizar trabajos de investigación. En estas situaciones, terminaba sucediendo que algunos estudiantes inmigrantes no habían conseguido un grupo al cual incorporarse, por lo cual los mismos profesores les pedían que integraran un grupo. Así, comenzaban vínculos de estudio que, en muchos casos, se convertían en amistades.

A partir de ello, es posible interpretar mejor los hallazgos de Beltrán y La Serna (2011), es decir, explicar por qué la brecha de rendimiento académico entre los estudiantes limeños y los inmigrantes se reduce entre el segundo y tercer año de estudios. Así, los primeros años, en los cuales los estudiantes inmigrantes evidenciaban un menor rendimiento académico que los alumnos limeños, constituyen el período de búsqueda de estrategias para mejorar el rendimiento académico que debían realizar los alumnos provenientes de provincias distintas a Lima. Se trata de un período en el cual los estudiantes 
inmigrantes probaban estrategias de estudio e iban descartando las que no funcionaban; paralelamente, iban construyendo redes de compañeros con los cuales el estudio en grupo era eficaz para el proceso de aprendizaje y la mejora del rendimiento académico.

Es evidente que la mayoría de desafíos académicos que enfrentaron y superaron los estudiantes inmigrantes también son enfrentados por sus pares limeños durante el tránsito del colegio a la universidad. Entre dichos desafíos, se encuentran el estudio memorístico, la prevalencia de estudio individual, la preparación con poca anticipación para las evaluaciones, el desfase entre los niveles de dificultad escolar y universitario en matemáticas, y el débil dominio del idioma inglés. Sin embargo, la presente investigación se ha enfocado en la manera que los inmigrantes experimentan estos desafíos, debido a que sus pares de Lima suelen disponer de recursos adicionales para enfrentarlos (redes sociales, acompañamiento directo y soporte económico familiar, etc.). Ahí se encuentra el origen de la brecha inicial de rendimiento académico entre limeños e inmigrantes que finalmente es superada.

\section{Conclusiones y recomendaciones}

A partir de las entrevistas realizadas, ha sido posible concluir que los estudiantes inmigrantes de la Universidad del Pacífico enfrentaron desafíos académicos muy retadores debido a los déficits que arrastraban de la etapa escolar: la costumbre de estudiar individualmente y tan solo unas pocas horas el día previo a la evaluación; la falta de conocimientos profundos de Matemáticas de nivel escolar y de ciertos saberes de Economía; y, finalmente, la falta de dominio del inglés. Ciertamente, conforme pasaban los semestres, los alumnos inmigrantes fueron probando y encontrando, por cuenta propia, estrategias para superar los desafíos que les imponía el currículo universitario y mejorar así su desempeño académico.

Entre las estrategias que aplicaron los estudiantes inmigrantes, destacan el incremento de las horas de estudio, así como la preparación con varios días de anticipación para las evaluaciones de los cursos más difíciles. Sin embargo, la estrategia más importante para mejorar su rendimiento académico fue cambiar el método de estudio individual y memorístico que arrastraban de la etapa escolar. Precisamente, la mayoría de entrevistados descubrió que la estrategia que mejor funcionaba era estudiar en grupos, a través de los cuales intercambiaban ideas y complementaban sus saberes. De este modo, se preparaban mejor para las evaluaciones.

La falta de redes en la ciudad de Lima determinó que los estudiantes inmigrantes demoraran en identificar un grupo de compañeros con los cuales resultaba productivo estudiar en grupo. Así, los primeros semestres constituyeron una etapa de "ensayo y error" tanto para identificar las estrategias de aprendizaje que funcionaran mejor para mejorar su rendimiento académico, como para ir construyendo redes de compañeros con los cuales resultaba efectivo estudiar. 
A partir de las conclusiones previas, es posible plantear algunas recomendaciones, con la cautela que implica extenderlas a otras universidades diferentes de aquella donde fue realizada la investigación. En primer lugar, no conviene dejar que la identificación de estrategias para enfrentar los desafíos académicos sea un proceso de "ensayo y error". Más bien, se requiere de un rol más activo de las universidades. Por ejemplo, la menor profundidad de los conocimientos escolares que los estudiantes de provincias aseguran sufrir puede ser compensada con un sistema de tutoría. Ciertamente, este acompañamiento debería abarcar diversos frentes. Para empezar, está el frente netamente académico; en este, es clave que los tutores apoyen a los estudiantes a nivelarse en aquellos contenidos en los que presentan algún déficit. Incluso, conviene considerar la posibilidad de brindar clases de refuerzo de nivel preuniversitario o cursos de comprensión lectora en inglés. En este caso, se requiere de tutores muy sólidos académicamente, que podrían ser estudiantes destacados, jefes de prácticas o profesores.

El sistema de acompañamiento también debería estar dirigido a brindar orientación que permita superar los desafíos de sociabilidad que enfrentan los estudiantes de provincias al migrar. Dicho acompañamiento debería enfocarse, en especial, en reconstruir sus redes sociales. Es importante reconocer que estos últimos tipos de apoyo requieren de tutores con perfil de psicólogo o trabajador social. No está de más considerar aplicar la figura denominada "hermano mayor", mediante la cual estudiantes de provincias que acaban de terminar sus estudios, o están por acabarlos, comparten sus experiencias y acompañan en el proceso de inserción a la vida universitaria a quienes recién ingresan a la universidad. Además, se requieren políticas organizacionales que rompan el hermetismo que caracteriza a los grupos de los limeños y que faciliten la integración de estos con los inmigrantes.

Por último, el presente estudio evidencia la importancia de acudir a métodos mixtos en la investigación educativa, tal como lo hizo aquel que condujo al presente artículo. Así, la investigación que originó el presente artículo acudió a entrevistas a profundidad, lo cual permitió interpretar y entender mejor los resultados que había arrojado un análisis cuantitativo previo.

\section{Agradecimientos}

Deseo agradecer la valiosa orientación recibida de parte del Dr. Martín Benavides Abanto en la elaboración de la presente investigación, así como a la Dra. Silvana Vargas Winstanley por sus enriquecedores comentarios a los avances de la investigación. 


\section{Referencias bibliográficas}

Araujo, K., y Martuccelli, D. (2010) La individuación y el trabajo de los individuos. Educação e Pesquisa, São Paulo, (36), 77-91. Recuperado de http://www.scielo.br/pdf/ep/v36nspe/v36nspea07.pdf

Ausubel, D. (1976). Psicología educativa. Un punto de vista cognoscitivo. México: Editorial Trillas.

Barrère, A. (2016). La relación educativa bajo la prueba de las actividades juveniles: Los nuevos desafíos profesionales. Propuesta Educativa, 25(46), 75-83. http:// www.propuestaeducativa.flacso.org.ar/archivos/articulos/56.pdf

Becerra, A. M., La Serna, K. (2016). Diseño curricular por competencias: Un enfoque para carreras de campo económico-empresarial. Lima: Universidad del Pacífico.

Beltrán, A., y La Serna, K. (2011). ¿Qué factores explican la evolución del rendimiento académico universitario? Un estudio de caso en la Universidad del Pacífico. En K. La Serna (Ed.), Retos para el aprendizaje: de la educación inicial a la universidad (pp. 251-322). Lima: Universidad del Pacífico.

Biggs, J. (2006). Calidad del aprendizaje universitario ( $2^{\mathrm{a}}$ ed.). Madrid: Narcea S.A. ediciones.

Cuenca, R. (2015). Democratización del acceso y la precarización del servicio. La masificación universitaria en el Perú, una introducción. En R. Cuenca (Ed.), La educación universitaria en el Perú: Democracia, expansión y desigualdades (pp. 9-17). Lima: Instituto de Estudios Peruanos.

García, M., y Medina, M. S. (2011). Factores que influyeron en el proceso de integración a la Universidad Católica y en el rendimiento académico de los alumnos que ingresaron en el 2014-I procedentes delos diferentesdepartamentos de Perú (tesis de maestría). Universidad Pontificia Universidad Católica del Perú (PUCP), Lima, Perú. http://tesis.pucp.edu.pe/repositorio/bitstream/ handle/123456789/1233/GARCIA_MAGALLY_MEDINA_MARIA_ FACTORES_INFLUYERON.pdf?sequence $=1$

Hernández, R., Fernández, C., y Baptista, P. (2010). Metodología de la investigación ( $5^{\mathrm{a}} \mathrm{Ed}$.). México: McGraw Hill.

Instituto Nacional de Estadística e Informática (1997). I Censo Universitario 1996: Síntesis estadística. Lima: Dirección Técnica de Censos y Encuestas del Instituto Nacional de Estadística e Informática.

Instituto Nacional de Estadística e Informática y Asamblea Nacional de Rectores (2011). II Censo universitario 2010. Principales resultados. Lima: Instituto Nacional de Estadística e Informática. Recuperado de http:// repositorio.minedu.gob.pe/bitstream/handle/123456789/865/503.\%20 II\%20Censo\%20Nacional\%20Universitario\%202010\%20Principales\%20 resultados.pdf? sequence $=1$ \&isAllowed $=y$ 
Johnson, D., Johnson, R., y Holubec, E. (1999) El aprendizaje cooperativo en el aula. Trad. de Gloria Vitale. Buenos Aires: Editorial Paidós SAICF.

La Serna, K. (2011). El producto del aprendizaje: El rendimiento académico. En K. La Serna (Ed.), Retos para el aprendizaje: de la educación inicial a la universidad (pp. 19-103). Lima: Centro de Investigación de la Universidad del Pacífico.

López, M., y López, A. (2013). Los enfoques de aprendizaje. Revisión conceptual y de investigación. Revista Colombiana de Educación, Bogotá, 64, 131-153. Recuperado de http://www.scielo.org.co/pdf/rcde/n64/n64a06.pdf

Martuccelli, D. (2004). Lo intercultural ante la prueba de la dinámica entre exclusión e integración social. Revista CIDOB d'Afers Internacionals, (66-67), 53-68. Recuperado de https://www.cidob.org/es/media2/ publicacions/afers/66_67/martucelli_cast

Pereyra-Elías, R., Ocampo-Mascaró, J., Silva-Salazar, V., Vélez-Segovia, E., Da Costa-Bullón, D., Toro-Polo, L. M., y Vicuña-Ortega, J. (2010). Prevalencia y factores asociados con síntomas depresivos en estudiantes de Ciencias de la Salud de una universidad privada de Lima. Revista Peruana de Medicina Experimental y Salud Pública, 27(4), 520-526. Recuperado de https://www.redalyc.org/articulo.oa?id=36318511005

Rama, C. (2005). La política de educación superior en América Latina y el Caribe. Revista de la Educación Superior, 134(2), 47-62. Recuperado de http://www.redalyc.org/pdf/604/60411920005.pdf

Solé, I., y Coll, C. (1998). Los profesores y la concepción constructivista. El constructivismo en aula ( $8^{\mathrm{a}}$ ed.). Barcelona: Editorial Graó.

Tang, K. C. C. (1993). Spontaneous collaborative learning: A new dimension in student learning experience? Higher Education Research and Development, 12(2), 115-130. 
